\title{
Pitfalls of prioritizing cost-effectiveness in the assessment of medical innovation: A case study of robotic-assisted prostatectomy in Ontario
}

\author{
Christopher J.D. Wallis MD, $\mathrm{PhD}^{1}$; Allan S. Detsky MD, $\mathrm{PhD}^{2}$ \\ ${ }^{1}$ Institute of Health Policy, Management \& Evaluation and Division of Urology, Department of Surgery, University \\ of Toronto; ${ }^{2}$ Institute of Health Policy, Management \& Evaluation and Department of Medicine, University of \\ Toronto; Department of Medicine, Mount Sinai Hospital and University Health Network; Toronto, ON, Canada
}

Cite as: Can Urol Assoc J 2017 Dec. 1; Epub ahead of print. http://dx.doi.org/10.5489/cuaj.5068

Published online December 1, 2017

***

I'm sure all readers of $C U A J$ have deliberated, and quite a few of us fretted, over articles and blogs such as the one in the Globe and Mail from this past summer entitled, "The fight over robots in the operating room.” At the heart of the controversy was a report from Health Quality Ontario on the fate of robotic-assisted radical prostatectomy in the province. In this CUAJ Guest Editorial, Chris Wallis and Allan Detsky from the Institute of Health Policy, Management \& Evaluation at the University of Toronto shed some much needed light on technology assessment.

$$
\text { - D. Robert Siemens, MD, FRCSC, CUAJ Editor-in-Chief }
$$

In 2010, the Ontario Ministry for Health and Long-term Care formed Health Quality Ontario (HQO). Part of HQO's mandate is to provide recommendations regarding the government's funding for health services and medical devices. Most recently, HQO undertook a Heath Technology Assessment (HTA) of robotic-assisted radical prostatectomy (RARP) ${ }^{1}$. In this Commentary, we use this example to highlight potential pitfalls of prioritizing cost-effectiveness in the application of HTA to the exclusion of other considerations, especially when estimates of cost-effectiveness are driven more by modelling than data.

HTA may be difficult to perform for many reasons. First, conclusions regarding clinical effectiveness may lack internal or external validity due to the use of non-randomized study designs, inappropriate comparators, problematic endpoints or narrow patient samples ${ }^{2}$. Second, there may be significant uncertainty about cost-effectiveness driven by difficulty in estimating cost (such as allocation of overhead) or measurement of quality of life. Third, because the models depend on extensive assumptions, their results are easily influenced by bias. However, even in the presence of adequate clinical and economic data, other factors including societal context and values, and the ability to negotiate price, have been shown to affect decisions about pharmaceutical coverage in the United Kingdom, Canada, and Australia ${ }^{2}$. The HQO analysis of RARP exemplifies some of these pitfalls. 
Despite abundant observational data, for reasons of internal validity, the HQO clinical effectiveness review appropriately considers only the single published randomized controlled trial which showed minimal difference between RP and RARP ${ }^{3}$. However, basing a firm HTA recommendation on a single study requires it to be considered incontrovertible and definitive, a standard that this study does not reach. First, rather than truly comparing one modality to another, this study compared Surgeon A performing open radical prostatectomy with Surgeon B performing RARP. In addition to well recognized differences in innate surgical skill, surgical experience is strongly associated with surgical outcomes ${ }^{4}$. While the study's open Surgeon A had more than 15 years and 1500 cases of experience prior to the trial initiation, the robotic Surgeon B had 2 years and 200 cases of experience. Thus, the generalizability of the exposure assessed is questionable. Second, the natural history of prostate cancer and of functional recovery following surgery is protracted. Therefore, assessment of outcomes at 12-weeks is too early to be meaningful, as the study's authors acknowledge ${ }^{3}$. Thus, the validity of the outcome used is questionable.

The HQO report then describes an elegant original Markov model used to make a judgement about the economic attractiveness of RARP. However, building a cost-effectiveness model by beginning with the assumption of no difference in the clinical outcomes of interest, which this report does, is futile. The denominator in the incremental cost-effectiveness ratio will, by definition, be zero and thus the ratio will approach infinity. In order to prevent this from happening here, the authors of the HQO report used very tiny differences in utility between therapies to reflect observed differences in pain during the recovery period, but again doing so only ensured that the denominator would be miniscule. A previous review of pharmaceutical funding recommendations in Ontario showed that cost-effectiveness estimates contributed substantially to the debate only in cases evaluating innovative drugs (those that offer clinically significant benefit with an increased cost) and was not helpful for "me-too" or generic drugs 5 . For drugs that had no incremental clinical benefit, as was assumed about RARP here, only cost estimates were needed, and most of these did not require sophisticated modelling. Further, the HQO report used an arbitrarily chosen threshold of economic attractiveness, an approach with other well-known problems ${ }^{6}$.

Additionally, the report's authors explicitly excluded patient involvement, concluding that "direct patient engagement would add relatively little new information” despite conceding

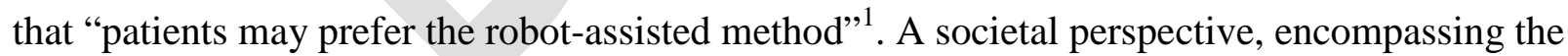
views of patients, would provide both a more robust methodology and more informative result ${ }^{8}$.

Finally, the methodology developed for assessment of pharmaceuticals may not be appropriate for surgical techniques. While breakthroughs in medical treatment necessitate writing a different prescription, innovation in surgical practice is an iterative process requiring a significant learning curve. HTA ought to consider "engendering innovation" in addition to the immediate clinical benefits of the technology ${ }^{7}$. Across nearly every industry, there is an increasing role for mechanization and robotics. In the past decade, much of the innovation in surgery has centered on the development and refinement of robotic equipment. Indeed, robotic 
surgery is not about differing approaches to individual operations but rather about a new surgical platform. This platform has allowed further surgical innovation, both within prostate cancer surgery and more broadly, that may allow for minimally invasive approaches to operations which previously required open surgery, shorter training periods required to achieve proficiency, and potentially improved ergonomics for surgeons that may allow for increased productivity or longer careers. Most importantly, jurisdictions that discourage innovation threaten their ability to attract, train, and recruit top surgeons, and thus compromise the quality of the medical workforce, which ought to be an important consideration in any HTA.

The report closes by recommending that the government of Ontario not pay the incremental non-fixed costs of RARP (with an estimated total annual budget impact that ranges from $\$ 0.4$ to $\$ 1.9$ million $C A D^{1}$, a small fraction of the total annual health care expenditures of $\$ 54$ billion), while acknowledging that the fixed costs of the machines have entirely been paid for by philanthropic donations (thus far). It stops there, making no recommendation about the way forward. While Ontario has, in part, successfully contained hospital expenditures by limiting the diffusion of unproven medical technologies in the past, we wonder whether such a definite pronouncement here appears myopic and, thus, misplaced.

We believe that this example of HTA demonstrates that an excessively limited perspective that inappropriately relies on a premature estimate of cost-effectiveness can lead to a short- sighted recommendation. The model was sophisticated; the analysis of the technology was not. We are not saying the report should have simply recommended paying for RARP, but rather that a more informative analysis would consider patients' perspectives, the ongoing evolution of robotics in medicine, and the impact of policy on recruiting and retaining human resources. In doing so it would provide a framework for innovation, development and evaluation of this technology which may or may not prove to be of value for Canadians in the future. 


\section{References}

1. Ontario HQ. Robotic surgical system for radical prostatectomy: a health technology assessment. Ont Health Technol Assess Ser [Internet]. 2017;17(11):1-172.

2. Clement FM, Harris A, Li JJ, Yong K, Lee KM, Manns BJ. Using effectiveness and costeffectiveness to make drug coverage decisions: a comparison of Britain, Australia, and Canada. JAMA : the journal of the American Medical Association. 2009;302(13):14371443.

3. Yaxley JW, Coughlin GD, Chambers SK, et al. Robot-assisted laparoscopic prostatectomy versus open radical retropubic prostatectomy: early outcomes from a randomised controlled phase 3 study. Lancet. 2016;388(10049):1057-1066.

4. Birkmeyer JD, Stukel TA, Siewers AE, Goodney PP, Wennberg DE, Lucas FL. Surgeon volume and operative mortality in the United States. The New England journal of medicine. 2003;349(22):2117-2127.

5. PausJenssen AM, Singer PA, Detsky AS. Ontario's formulary committee: how recommendations are made. Pharmacoeconomics. 2003;21(4):285-294.

6. Detsky AS, Laupacis A. Relevance of cost-effectiveness analysis to clinicians and policy makers. JAMA : the journal of the American Medical Association. 2007;298(2):221-224.

7. Turchetti G, Spadoni E, Geisler E. Health Technology Assessment: Evaluation of Biomedical Innovative Technologies. IEEE Engineering in Medicine and Biology Magazine. Vol May/June 20102010:70-76. 